\title{
Schema-based Scheduling of Event Processors and Buffer Minimization for Queries on Structured Data Streams
}

\author{
Christoph Koch ${ }^{\dagger, *} \quad$ Stefanie Scherzinger ${ }^{\ddagger} \quad$ Nicole Schweikardt ${ }^{\natural} \quad$ Bernhard Stegmaier ${ }^{\sharp}$ \\ †: Technische Universität Wien, Vienna, Austria, Email: koch@dbai.tuwien.ac.at \\ †: Technische Universität Wien, Vienna, Austria, Email: scherzinger@wit.tuwien.ac.at \\ घ: Humboldt Universität zu Berlin, Berlin, Germany, Email: schweikardt@informatik.hu-berlin.de \\ \#: Technische Universität München, Munich, Germany, Email: bernhard.stegmaier@in.tum.de
}

\begin{abstract}
We introduce an extension of the XQuery language, FluX, that supports event-based query processing and the conscious handling of main memory buffers. Purely event-based queries of this language can be executed on streaming XML data in a very direct way. We then develop an algorithm that allows to efficiently rewrite XQueries into the event-based FluX language. This algorithm uses order constraints from a DTD to schedule event handlers and to thus minimize the amount of buffering required for evaluating a query. We discuss the various technical aspects of query optimization and query evaluation within our framework. This is complemented with an experimental evaluation of our approach.
\end{abstract}

\section{Introduction}

XML is the preeminent data exchange format on the Internet. Stream processing naturally bears relevance in the data exchange context (e.g., in e-commerce). An increasingly important data management scenario is the processing of XQueries on streams of exchanged XML data. While the weaknesses of XML as a semistructured data model have been observed time and again (cf. e.g. [1]), XQuery on XML streams can be seen as the prototypical instance of the problem of queries on structured (vs. flat tuple) data streams.

Query engines for processing streams are naturally main-memory-based. Conversely, in some ef-

\footnotetext{
* Work support by project Z29-N04 of the Austrian Science Fund (FWF)

Permission to copy without fee all or part of this material is granted provided that the copies are not made or distributed for direct commercial advantage, the VLDB copyright notice and the title of the publication and its date appear, and notice is given that copying is by permission of the Very Large Data Base Endowment. To copy otherwise, or to republish, requires a fee and/or special permission from the Endowment.

Proceedings of the 30th VLDB Conference, Toronto, Canada, 2004
}

forts towards developing main-memory XQuery engines whose original emphasis was not on stream processing (e.g., BEA's XQRL [9]), it was observed that it is worthwhile to build such systems using stream processing operators.

The often excessive need for buffers in current main memory query engines causes a scalability issue that has been identified as a significant research challenge $[14,13,16,7,4]$. While the efficient evaluation of XPath queries on streams has been worked on extensively in the past (here, state-of-the-art techniques use very little main memory) $[2,5,10,11]$, not much work has been done on efficiently processing XQuery on streams. The nature of XQuery, as a data-transformation query language entirely different from node-selecting XPath, requires new techniques for dealing with (and reducing) main memory buffers. State-of-the-art XQuery engines consume main memory in large multiples of the actual size of input XML documents [14].

Several recent projects have addressed XQuery on streams using transducer networks $[13,16]$. Automata-based techniques are usually quite elegant but are hard to compare or integrate with other approaches and usually do not generalize to real-world query languages such as (full) XQuery with their great expressive power and all their odd features and artifacts of the standardization process. One approach [14] towards addressing the problem of reducing main memory consumption in an engine for full XQuery aims at reducing the amount of data buffered in main memory by pre-filtering the data read from the stream with the paths occurring in the query. However, for realworld XQueries, the need for substantial main memory buffers cannot be avoided in general.

An important goal is thus to devise a well-principled machinery for processing XQuery that is parsimonious with resources and allows to minimize the amount of buffering. Such machinery needs to be based on intermediate representations of queries that are syntactically close to XQuery and has to allow for an algebraic approach to query optimization, with buffering 
as an optimization target. This is necessary to allow for both extensibility and the leverage of a large body of related earlier work done by the database research community. However, to our knowledge, no principled work exists on query optimization in the framework of XQuery (rather than automata) for structured data streams (such as XML, but unlike flat tuple streams) which honors the special features of stream processing. Moreover, no framework for optimizing queries on structured data streams exists that captures the spirit of stream processing and allows for query optimization using schema information. (However, there are XQuery algebras meant for conventional query processing $[17,8]$, and there is work on applying them in the streaming context [7]. Moreover, the problem of optimizing XQueries using a set of constraints holding in the XML data model - rather than a schema - was addressed in [6].)

In this paper, we attempt to improve on this situation. We introduce a query language, FluX, which extends XQuery by a new construct for event-based query processing called process-stream. FluX motivates a very direct mode of query evaluation on data streams (similar to query evaluation in XQRL [9]), and provides a strong intuition for what main memory buffers are needed in which queries. This allows for a strongly "buffer-conscious" mode of query optimization. The main focus of this paper is on automatically rewriting XQueries into event-based FluX queries and at the same time optimizing (reducing) the use of buffers using schema information from a DTD.

Consider the following XQuery $Q$ in a bibliography domain, taken from the XML Query Use Cases [18] (XMP Q3):

\section{<results> \\ $\{$ for $\$ b$ in $\$ R 0 O T / b i b /$ book return $\langle$ result $>\{\$ \mathrm{~b} /$ title $\}\{\$ \mathrm{~b} /$ author $\}</$ result $>\}$ $\langle/$ results $\rangle$}

For each book in the bibliography, this query lists its title(s) and authors, grouped inside a "result" element. Note that the XQuery language requires that, within each book, all titles are output before all authors.

The DTD

$<$ !ELEMENT bib (book)*>

$<$ !ELEMENT book (title|author)*>

specifies that each book node may have several title and several author children. A priori, no order among these items is inferable from the given DTD. To implement this query, we may output the title children inside a book node as soon as they arrive on the stream. However, the output of the author children needs to be delayed (using a memory buffer) until we reach the closing tag of the book node (at that time, no further title nodes may be encountered). Then we may flush the buffer of author nodes, empty it, and later refill it with the author nodes from the next book.

We thus only need to buffer the author children of one book node at a time, but not the titles. Current main memory query engines do not exploit this fact, and rather buffer either the entire book nodes or, as an optimization [14], all title and all author nodes of book. Previous frameworks for evaluating or optimizing XQuery do not provide any means of making this seeming subtlety explicit and reasoning about it.

The process-stream construct of FluX allows to express precisely the mode of query execution just described. XQuery $Q$ is then phrased as a FluX query as follows:

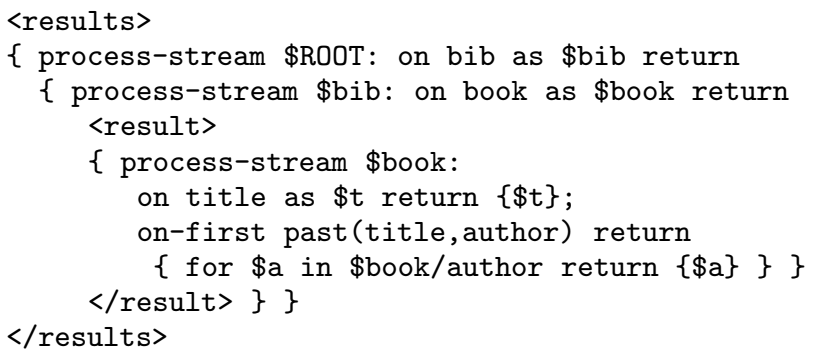

A process-stream \$x expression consists of a number of handlers which process the children of the XML tree node bound by variable $\$ \mathrm{x}$ from left to right. An "on a" handler fires on each child labeled "a" visited during such a traversal, executing the associated query expression. In the process-stream \$book expression above, the on-first past(title, author) handler fires exactly once as soon as the DTD implies for the first time that no further author or title node can be encountered among the children of \$book. (As observed above, in the given, very weak DTD, this is the case only as soon as the last child of \$book has been seen.) In the query associated with the on-first past (title, author) handler, we may freely use paths of the form \$book/author or \$book/title, because such paths cannot be encountered anymore and we may assume that the query engine has already buffered all matches of these paths for us. It is a feasible task for the query engine to buffer only those paths that the query actually employs (see also [14]).

We call a query safe for a given DTD if, informally, it is guaranteed that XQuery subexpressions (such as the for-loop in the query above) do not refer to paths that may still be encountered in the stream. The above FluX query is safe: The for-expression employs the \$book/author path, but is part of an on-first handler that cannot fire before all author nodes relative to \$book have been seen.

If the path \$book/author was replaced by, say, $\$$ book/price and the DTD production for book were

<!ELEMENT book ((titlelauthor)*,price)>

then the FluX query above would not be safe. In that case, on the firing of on-first past (title, author), the buffer for \$book/price items would still be empty and the query result would be incorrect.

Query $Q$ can be processed more efficiently with the schema used in the XML Query Use Cases, 
$<$ !ELEMENT bib (book)*>

$<$ !ELEMENT book (title, (author+|editor+), publisher,price)>

Here, no buffering is required to execute our query because the DTD asserts that for each book, the title occurs strictly before the authors (we denote this as $\operatorname{Ord}_{\text {book }}$ (title, author), called an order constraint). We may phrase our query in FluX so as to directly copy titles and authors to the output as they arrive on the input stream. No data items need to be buffered.

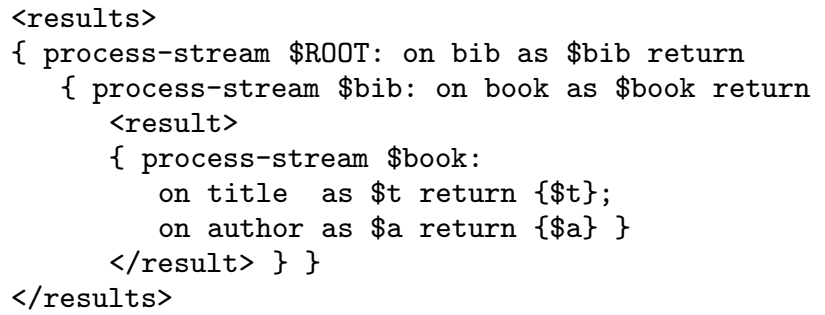

The contributions of this paper are as follows.

- We introduce the FluX query language, which extends XQuery by the natural stream processing construct discussed above.

- We define the safe FluX queries (under a given DTD), which are those FluX queries in which XQuery subexpressions have the usual semantics (i.e., are never executed before the data items referred to have been fully read from the stream and may be assumed available in main memory buffers).

- We present an algorithm that schedules XQueries on streams using DTDs and transforms them into optimized FluX queries.

- We discuss the realization of query engines for FluX and the runtime buffer management.

- We have built a prototype FluX query engine which we evaluate by means of a number of experiments.

This is, to our knowledge, the first work on optimizing XQuery using schema constraints derived from DTDs $^{1}$. A main strength of the approach taken in this paper is its extensibility, and even though space limitations require us to restrict our discussion to a (powerful) fragment of XQuery, our results can be generalized to even larger fragments. In our discussion at the end of the paper, we will also lay the foundations for algebraic optimization of queries using further information from the schema.

This paper is structured as follows. We start with basics on DTDs and regular languages in Section 2 . Section 3 defines the query languages considered in this paper: Section 3.1 specifies an XQuery fragment. Based on this, Section 3.2 defines the FluX language, and Section 3.3 singles out the safe FluX queries. Section 4 presents our algorithms for translating XQuery

\footnotetext{
${ }^{1}$ To simplify presentation we restrict ourselves to DTDs, but the required information could also be derived from XML Schemata.
}

into a particular normal form (Section 4.1) and for transforming this normal form into FluX (Section 4.2). Some examples of this transformation are given in Section 4.3. In Section 5 we discuss the implementation of our prototype system and the actual handling of buffers during query evaluation. In Section 6, we present our experiments, and we conclude with a discussion in Section 7.

\section{Preliminaries}

For simplicity of exposition, we consider the fragment of XML without attributes as our data model. Note that this is no substantial restriction, since attributes can be handled in the same way as subelements.

We focus on valid documents, i.e. documents conforming to a given document type definition (DTD).

Let $\Sigma$ be a set of symbols (or tag names). A DTD is an extended context free grammar over $\Sigma$. DTDs are local tree grammars [15], i.e. without competing nonterminals to the left-hand sides of productions, so each production in a DTD is unambiguously identified by a tag name in $\Sigma$.

Let $\rho$ be a regular expression and let $\operatorname{symb}(\rho)$ be the set of atomic symbols that occur in $\rho$. By $L(\rho)$ we denote the language defined by $\rho$, i.e., the set of words over $\operatorname{sym} b(\rho)$ that are recognizable by $\rho$. Given a word $w$, let $w_{i}$ denote its $i$-th symbol. We define a binary relation $\operatorname{Ord}_{\rho} \subseteq \Sigma \times \Sigma$ such that for $a, b \in \Sigma$,

$$
\operatorname{Ord}_{\rho}(a, b): \Leftrightarrow \nexists w \in L(\rho): w_{i}=b \wedge w_{j}=a \wedge i<j .
$$

That is, $\operatorname{Ord}_{\rho}(a, b)$ holds if there is no word in $L(\rho)$ in which a symbol $a$ is preceded by a symbol $b$. (All $a$ symbols occur before all $b$ symbols.) We refer to a constraint of the form $\operatorname{Ord}_{\rho}(a, b)$ as an order constraint.

Example 2.1 Let $\rho=\left(a^{*} \cdot b \cdot c^{*} \cdot\left(d \mid e^{*}\right) \cdot a^{*}\right)$. Then, $\operatorname{Ord}_{\rho}(b, c), \operatorname{Ord}_{\rho}(c, d)$, and $\operatorname{Ord}_{\rho}(c, e)$, but $\neg \operatorname{Ord}_{\rho}(a, c)$. $\operatorname{Ord}_{\rho}$ is transitive, so we also have e.g. $\operatorname{Ord}_{\rho}(b, d)$.

DTDs have the nice property that regular expressions appearing in the right-hand sides of productions are one-unambiguous. This guarantees that an equivalent deterministic finite automaton can be computed in polynomial - even quadratic - time [3]. One can show the following [12]:

Proposition 2.2 Given a regular expression $\rho$ from a DTD, Ord $\rho$ can be computed in time $O\left(|\rho|^{2}\right)$.

Let $\rho$ be a regular expression and let $S \subseteq \Sigma$. Then, for each word $u=u_{1} \ldots u_{n} \in \operatorname{sym} b(\rho)^{*}$,

$$
\begin{aligned}
& \text { Past }_{\rho, S}(u): \Leftrightarrow \\
& \quad \forall w \in \operatorname{symb}(\rho)^{*}: u w \in L(\rho) \rightarrow \nexists i: w_{i} \in S, \\
& \text { first-past }_{\rho, S}(u): \Leftrightarrow \\
& \text { Past }_{\rho, S}(u) \wedge\left(n>0 \rightarrow \neg \text { Past }_{\rho, S}\left(u_{1} \ldots u_{n-1}\right)\right) .
\end{aligned}
$$

Intuitively, when processing a word $u w \in L(\rho)$ from left to right, if first-past $_{\rho, S}(u)$ holds, then the reading of the last symbol of $u$ is the earliest possible time at which we know that none of the symbols in $S$ can be seen anymore until the end of the word $u w$. 


\section{Query Language}

In this section, we define the syntax and semantics of the FluX query language, which extends an XQuery fragment, denoted as $\mathrm{XQuery}^{-}$, by a construct for event-based query processing.

Before defining FluX and XQuery ${ }^{-}$, we need some more notation. We write $\$ x, \$ y, \$ z, \ldots$ to denote variables that range over XML trees. In the following, we overload the meaning of variable $\$ x$ bound to an XML tree whose root is labeled $a$, by writing $\$ x$ when we actually mean the DTD production unambiguously identified by the element $a$. For example, if the DTD contains the rule $<$ !ELEMENT $a \rho^{a}>$ for a regular expression $\rho^{a}$, we write $\operatorname{Ord}_{\$ x}(c, d)$ instead of $\operatorname{Ord}_{\rho^{a}}(c, d)$, and we write $\operatorname{symb}(\$ x)$ instead of $\operatorname{symb}\left(\rho^{a}\right)$.

A fixed path is a sequence $a_{1} / \ldots / a_{n}$, where the $a_{i}$ are symbols from the DTD and $n \geq 1$. XPath expressions such as $a / * / b$, or $a / / b$ or $a[b]$ are excluded.

An atomic condition is either of the form $\$ x / \pi \operatorname{RelOp} s$, exists $\$ x / \pi$, or $\$ x / \pi$ RelOp $\$ y / \pi^{\prime}$, where $s$ is a string, $\pi$ and $\pi^{\prime}$ are fixed paths, and RelOp $\in\{=,<, \leq,>, \geq\}$. A condition is a Boolean combination (using "and", "or", "not", and "true") of atomic conditions.

\subsection{An XQuery Fragment: XQuery ${ }^{-}$}

Definition 3.1 (XQuery ${ }^{-}$) The XQuery fragment $\mathrm{XQuery}^{-}$is the smallest set consisting of expressions

1. $\epsilon$ (the empty query)

2. $s$ (output of a fixed string)

3. $\alpha \beta$ (sequence)

4. $\{$ for $\$ x$ in $\$ y / \pi$ return $\alpha\}$ (for-loop)

5. $\{$ for $\$ x$ in $\$ y / \pi$ where $\chi$ return $\alpha\}$ (conditional for-loop)

6. $\{\$ x / \pi\}$ (output of subtrees reachable from node $\$ x$ through path $\pi$ )

7. $\{\$ x\}$ (output of subtree of node $\$ x$ )

8. $\{$ if $\chi$ then $\alpha\}$ (conditional)

where $\pi$ is a fixed path, $s$ a fixed string, $\chi$ a condition, and $\alpha$ and $\beta$ are XQuery ${ }^{-}$expressions.

Indeed, XQuery ${ }^{-}$is very similar to (a fragment of) standard XQuery [17], but differs in how we treat fixed strings inside queries. For example, the string 〈hello〉 is valid in XQuery ${ }^{-}$, but not in standard XQuery. The query

\section{$\langle$ result $>\{\$$ ROOT/bib/book $\}</$ result $>$}

is understood in standard XQuery as a "result" node with an embedded query to produce its children. In the present paper, the same query is read as a sequence of three queries which write the string $\langle$ result $\rangle$, the $/$ bib/book subtrees, and finally the string $\langle/$ result $\rangle$ to the output.
This, however, is only a subtlety which, on the one hand, is very convenient for obtaining our main results in Section 4 and which, on the other hand, as the following Proposition 3.2 shows, does not cause any problems. The alternative semantics of XQuery ${ }^{-}$ is the basis of optimizations used internally by the query engine. Users formulate input queries in standard XQuery and may assume the usual semantics.

Let $\llbracket Q \rrbracket_{X Q u e r y^{-}}(D)$ (resp., $\llbracket Q \rrbracket_{X Q u e r y}(D)$ ) denote the XML document stream produced by evaluating query $Q$ on document $D$ under our XQuery ${ }^{-}$semantics (resp., under the standard XQuery semantics [17]).

Proposition 3.2 Let $Q$ be an XQuery that parses as an XQuery ${ }^{-}$query. Then, for any input document $D$, $\llbracket Q \rrbracket_{X Q u e r y^{-}}(D)=\llbracket Q \rrbracket_{X Q u e r y}(D)$.

\subsection{Syntax and Semantics of FluX}

A simple expression is an XQuery ${ }^{-}$expression of the form $\alpha \beta \gamma$ where

- $\alpha$ and $\gamma$ are possibly empty sequences of strings and of expressions of the form " $\{$ if $\chi$ then $s\}$ ", where $\chi$ is a condition and $s$ is a string.

- $\beta$ is either empty, " $\{\$ u\}$ ", or " $\{$ if $\chi$ then $\{\$ u\}\}$ ", for some variable $\$ u$ and some condition $\chi$.

- if $\beta$ is of the form " $\{\$ u\}$ ", or " $\{$ if $\chi$ then $\{\$ u\}\}$ ", then no atomic condition that occurs in $\alpha \beta$ contains the variable $\$ u$.

For instance,

$$
<\mathrm{a}>\{\$ x\}</ \mathrm{a}>\{\text { if } \$ x / \mathrm{b}=5 \text { then }\langle\mathrm{b}>5</ \mathrm{b}\rangle\}
$$

is a simple expression, but $\{\$ x\}\{\$ y\}$ is not.

Definition 3.3 (FluX) The class of FluX expressions is the smallest set of expressions that are either simple or of the form

$$
s\{\text { process-stream } \$ y: \zeta\} s^{\prime}
$$

where $s$ and $s^{\prime}$ are possibly empty strings, $\$ y$ is a variable, and $\zeta$ is a list (where entries are separated by semicolons ";") of one or more event handlers. Each event handler is of one of the following two types:

1. (so-called "on-first" handler) on-first past $(S)$ return $\alpha$

where $S \subseteq \operatorname{symb}(\$ y)$ and $\alpha$ is an XQuery ${ }^{-}$expression

2. (so-called "on" handler)

on $a$ as $\$ x$ return $Q$

where $\$ x$ is a variable, $a$ is an element name in $\operatorname{symb}(\$ y)$, and $Q$ is a FluX expression.

We will use ps as a shortcut for process-stream, on-first past $(*)$ as an abbreviation for on-first past $(\operatorname{symb}(\$ y))$, and furthermore on-first past() in place of on-first past $(\emptyset)$. 
Some examples of FluX expressions, as well as an informal description of the FluX semantics, were already given in Section 1; further examples can be found in Section 4.3. In general, we evaluate an expression

$$
\{\text { process-stream } \$ y: \zeta\}
$$

as follows: An event-handling statement considers the children of the node currently bound by variable $\$ y$ as a list (or stream) of nodes and processes this list one node at a time. On processing a node $v$ with children $t_{1}, \ldots, t_{n}$, with the labels of $t_{i}$ denoted as label $\left(t_{i}\right)$, we proceed as follows. For each $i$ from 0 to $n+1$ (i.e., $n+2$ times), we scan the list of event handlers $\zeta=\zeta_{1} ; \ldots ; \zeta_{m}$ once from the beginning to the end. In doing so, we test for each event handler $\zeta_{j}$ whether its event condition is satisfied, in which case the event handler $\zeta_{j}$ "fires" and the corresponding query expression is executed:

- A handler "on $a$ as $\$ x$ return $Q$ " fires if $1 \leq i \leq$ $n$ and $\operatorname{label}\left(t_{i}\right)=a$.

- A handler "on-first past $(S)$ return $\alpha$ " fires if $0 \leq i \leq n$ and first-past ${ }_{\$ y, S}\left(\operatorname{label}\left(t_{1}\right) \ldots\right.$ label $\left.\left(t_{i}\right)\right)$ is true (i.e., for the first time while processing the children of $\$ y$, no symbol of $S$ can be encountered anymore) or if $i=n+1$ and this event handler has not fired in any of the previous $(n+1)$ scans.

In summary, it is well possible that several events fire for a single node, in which case they are processed in the order in which the handlers occur in $\zeta$. During the run on $t_{1}, \ldots, t_{n}$, each "on" handler may fire zero up to several times, while each "on-first" handler is executed exactly once.

For a FluX or XQuery ${ }^{-}$expression $Q$, let free $(Q)$ be the set of all free variables in $Q$, defined analogously to the free variables of a formula in first-order logic. That is, $\operatorname{free}(\{\$ x / \pi\})=\{\$ x\}$, and $\operatorname{free}(\{$ if $\chi$ then $\alpha\})$ consists of free $(\alpha)$ and the variables that appear in $\chi$. Further, free $(\{$ for $\$ x$ in $\$ y / \pi$ return $\alpha\})$ contains the variable $\$ y$ and the variables in free $(\alpha) \backslash\{\$ x\}$. Finally, free (\{process-stream $\$ y: \zeta\})$ consists of the variable $\$ y$, for each event handler in $\zeta$ of the form "on-first past $(S)$ return $\alpha$ " also of the variables in free $(\alpha)$, and likewise for each event handler in $\zeta$ of the form "on $a$ as $\$ x$ return $Q$ " of the variables in $\operatorname{free}(Q) \backslash\{\$ x\}$.

Note that expressions of the form " for $\$ x$ in $\$ y / a$ return $\alpha\}$ " and event handlers of the form "on $a$ as $\$ x$ return $Q$ " bind the variable $\$ x$, i.e., remove it from the free variables of the superexpressions.

A FluX query is a FluX expression in which all free variables except for the special variable \$ROOT corresponding to (the root of) the document are bound. That is, for a query $Q$ in FluX (resp., $\alpha$ in $\mathrm{XQuery}^{-}$) we require that free $(Q) \subseteq\{\$ \mathrm{ROOT}\}$ (resp., free $(\alpha) \subseteq$ $\{\$ R 00 T\}$ ).
As the following example shows, every XQuery ${ }^{-}$ query can be transformed into a FluX query in a straightforward way.

Example 3.4 Every XQuery ${ }^{-}$query $\alpha$ is equivalent to the FluX query

\section{$\{$ ps \$ROOT: on-first past $(*)$ return $\alpha\}$}

In Section 4 below we will show how, depending on a given DTD, this FluX query can be transformed into an equivalent FluX query that can be evaluated more efficiently.

By the size of an expression $Q$, denoted $|Q|$, we refer to the size of its string representation.

By the parent variable of (FluX or XQuery ${ }^{-}$) expression $\alpha$ in FluX query $Q$, denoted parent $\operatorname{Var}(\alpha)$, we refer to the variable bound by the nearest superexpression of $\alpha$, or \$ROOT if no such variable exists.

By the condition paths in $\alpha$, we refer to the set of paths $\$ x / \pi$ in a condition $\chi$ that occurs in $\alpha$.

For FluX or XQuery ${ }^{-}$expressions $\alpha$ and $\beta$ we write $\alpha \preceq \beta$ (resp., $\alpha \prec \beta$ ) to denote that $\alpha$ is a subexpression (resp., proper subexpression) of $\beta$. An XQuery $^{-}$subexpression $\alpha$ of a FluX expression $Q$ is called maximal if there is no XQuery ${ }^{-}$expression $\beta$ with $\alpha \prec \beta \preceq Q$. Note that a FluX query may contain several such maximal expressions.

Example 3.5 The maximal XQuery ${ }^{-}$subexpressions of the first FluX query from Section 1 are " $\{\$ \mathrm{t}\}$ " and

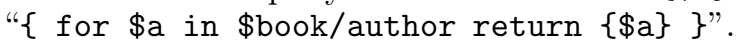

\subsection{Safe Queries}

We next define the notion of safety for FluX queries. Informally, a query is called safe for a given DTD if it is guaranteed that XQuery ${ }^{-}$subexpressions do not refer to paths that might still be encountered in an input stream compliant with the given DTD. For the precise definition we need the following notion.

The set of dependencies w.r.t. variable $\$ y$ in a FluX or XQuery ${ }^{-}$expression $\alpha$ is defined as

dependencies $(\$ y, \alpha):=$

$\{a \mid$ ex. a condition path $\$ y / a$ or $\$ y / a / \pi$ in $\alpha\} \cup$

$\{b \mid$ ex. $\$ u, \pi, Q$ s.t. $\pi$ starts with symbol $b$ and " $\{$ for $\$ u$ in $\$ y / \pi$ return $Q\} " \preceq \alpha\}$.

Definition 3.6 (safe queries) A FluX query $Q$ is called safe w.r.t. a given DTD if, and only if, for each subexpression " $\{\mathrm{ps} \$ y: \zeta\}$ " of $Q$, the following two conditions are satisfied:

1. For each handler "on-first past $(S)$ return $\alpha$ " in the list $\zeta$, the following is true:

- $\forall b \in$ dependencies $(\$ y, \alpha)$ we have: $b \in S$ or ex. $a \in S$ s.t. $\operatorname{Ord}_{\$ y}(b, a)$

- $\forall \$ z \in$ free $(\alpha)$ s.t. $\{\$ z\} \preceq \alpha$ or $\{\$ z / \pi\} \preceq \alpha$ (for some $\pi$ ) we have: $\$ z=\$ y$ and $\forall b \in \operatorname{symb}(\$ y)$ : $b \in S$ or ex. $a \in S$ s.t. $\operatorname{Ord}_{\$ y}(b, a)$. 


\author{
$\{$ for $\$ x$ in $\$ y / \pi$ where $\chi$ return $\beta\}$ \\ \{for $\$ x$ in $\$ y / \pi$ return $\{$ if $\chi$ then $\beta\}\}$ \\ $\frac{\{\$ y / \pi\}}{\{\text { for } \$ x \text { in } \$ y / \pi \operatorname{return}\{\$ x\}\}}$ \\ $\frac{\{\text { for } \$ x \text { in } \$ y / a / \pi \text { return } \beta\}}{\left\{\begin{array}{l}\text { for } \$ x_{0} \text { in } \$ y / a \text { return } \\ \left\{\text { for } \$ x \text { in } \$ x_{0} / \pi \text { return } \beta\right\}\end{array}\right\}}\left(\$ x_{0}\right.$ new $)$ \\ $\frac{\{\text { if } \chi \text { then }\{\text { for } \$ x \text { in } \$ y / \pi \text { return } \alpha\}\}}{\{\text { for } \$ x \text { in } \$ y / \pi \operatorname{return}\{\text { if } \chi \text { then } \alpha\}\}}$

$\frac{\{\text { if } \chi \text { then } \alpha \beta\}}{\{\text { if } \chi \text { then } \alpha\}\{\text { if } \chi \text { then } \beta\}}$
$\frac{\{\text { if } \chi \text { then }\{\text { if } \psi \text { then } \alpha\}\}}{\{\text { if }(\chi \text { and } \psi) \text { then } \alpha\}}$

Figure 1: Normal form rewrite rules. Each rule is always applied downwards, i.e., the expression above the line is replaced by the expression below the line.

2. For each handler "on $a$ as $\$ x$ return $\tilde{Q}$ " in the list $\zeta$, and for each maximal XQuery ${ }^{-}$subexpression $\alpha$ of $\tilde{Q}$, the following is true:

- $\forall b \in$ dependencies $(\$ y, \alpha)$ we have: $\operatorname{Ord}_{\$ y}(b, a)$

- if $\alpha=\tilde{Q}$ (note that according to Definition 3.3 $\alpha$ must then be simple), then for all $\$ u$ s.t. $\{\$ u\} \preceq \alpha$ we have: $\$ u=\$ x$.

It can be shown that this notion of safety is sufficient to ensure that main memory buffers are fully populated when they are accessed by a query, i.e., that a FluX query can be evaluated in a straightforward way on input streams compliant with the given DTD.

Examples of safe FluX queries can be found in Sections 1 and 4. (To be precise, all FluX queries occurring in this paper are safe.)

\section{Translating XQuery into FluX}

In this section we address the problem of rewriting a query of our XQuery fragment into an equivalent FluX query that employs as little buffering as possible. This rewriting proceeds in two steps: First, we transform the given $\mathrm{XQuery}^{-}$query into an equivalent query in XQuery ${ }^{-}$normal form (Section 4.1). Afterwards, depending on a given DTD, this normalized query is rewritten into an equivalent safe FluX query (Section 4.2). The FluX extensions manage the event based, streaming execution of the query. All subqueries exclusively working on buffered data are XQuery $^{-}$expressions.

\subsection{A Normal Form for XQuery ${ }^{-}$}

An XQuery ${ }^{-}$expression is transformed into normal form by rewriting (subexpressions of) it using the rules in Figure 1 until no further changes are possible.

In an XQuery ${ }^{-}$expression in normal form, the following three properties hold: (1) All paths except those inside conditionals are simple-step paths, i.e. of the form $\$ x / a$. (2) An expression in normal form does not contain any conditional for-loops, as the normalization process pushes conditionals inside the innermost for-loops. (3) For each subexpression of the form " $\{$ if $\chi$ then $\alpha\}$ ", $\alpha$ is either a fixed string or of the form " $\{\$ x\}$ " for some variable $\$ x$.

Theorem 4.1 The rule applications of Figure 1 can be implemented in such a way that the rewriting terminates for an input XQuery ${ }^{-}$expression $Q$ after $O(|Q|)$ rule applications with a unique result, the so-called normalization of $Q$, which is equivalent to $Q$.

Example 4.2 ([18], XMP, Q1) Consider the following XQuery $Q_{1}$ for books published by AddisonWesley after 1991, including their year and title.

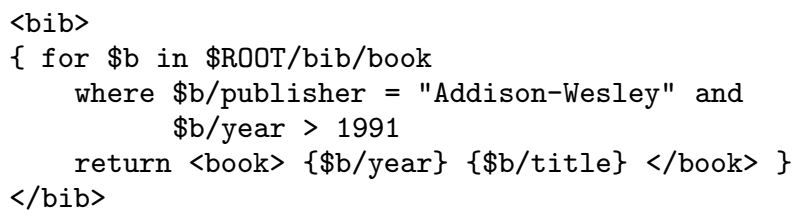

We abbreviate the where-condition in the above query as $\chi$. Then $Q_{1}$ has the following normalization $Q_{1}^{\prime}$ :

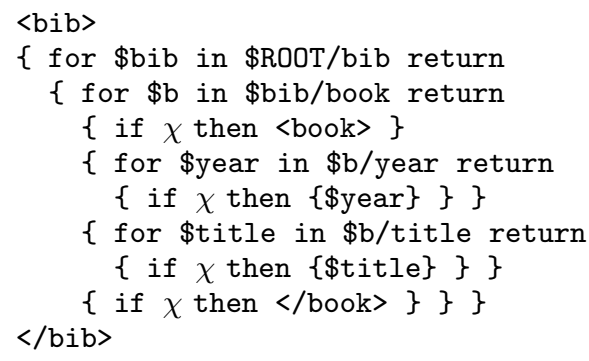

\subsection{Rewriting normalized XQuery ${ }^{-}$into FluX}

To formulate our main rewrite algorithm for transforming normalized XQuery ${ }^{-}$queries into equivalent, safe FluX queries, we need some further notation.

Let $\Sigma$ be the set of tag names occurring in the given DTD. Let $\perp$ denote the empty list. For a list $\zeta$ of event handlers, we inductively define the set $h \operatorname{sym} b(\zeta)$ of handler symbols for which an "on" handler or an "on-first" handler exists in $\zeta$ :

$$
\begin{gathered}
h \operatorname{symb}(\perp):=\emptyset \\
h \operatorname{symb}(\zeta ; \text { on } a \text { as } \$ x \operatorname{return} \alpha):=h \operatorname{symb}(\zeta) \cup\{a\} \\
h \operatorname{symb}(\zeta ; \text { on-first past }(S) \operatorname{return} \alpha):= \\
h \operatorname{symb}(\zeta) \cup S
\end{gathered}
$$

Our algorithm for recursively rewriting normalized XQuery ${ }^{-}$expressions into FluX is shown in Figure 2. Note that this algorithm uses order constraints and hence depends on the underlying DTD. Given 


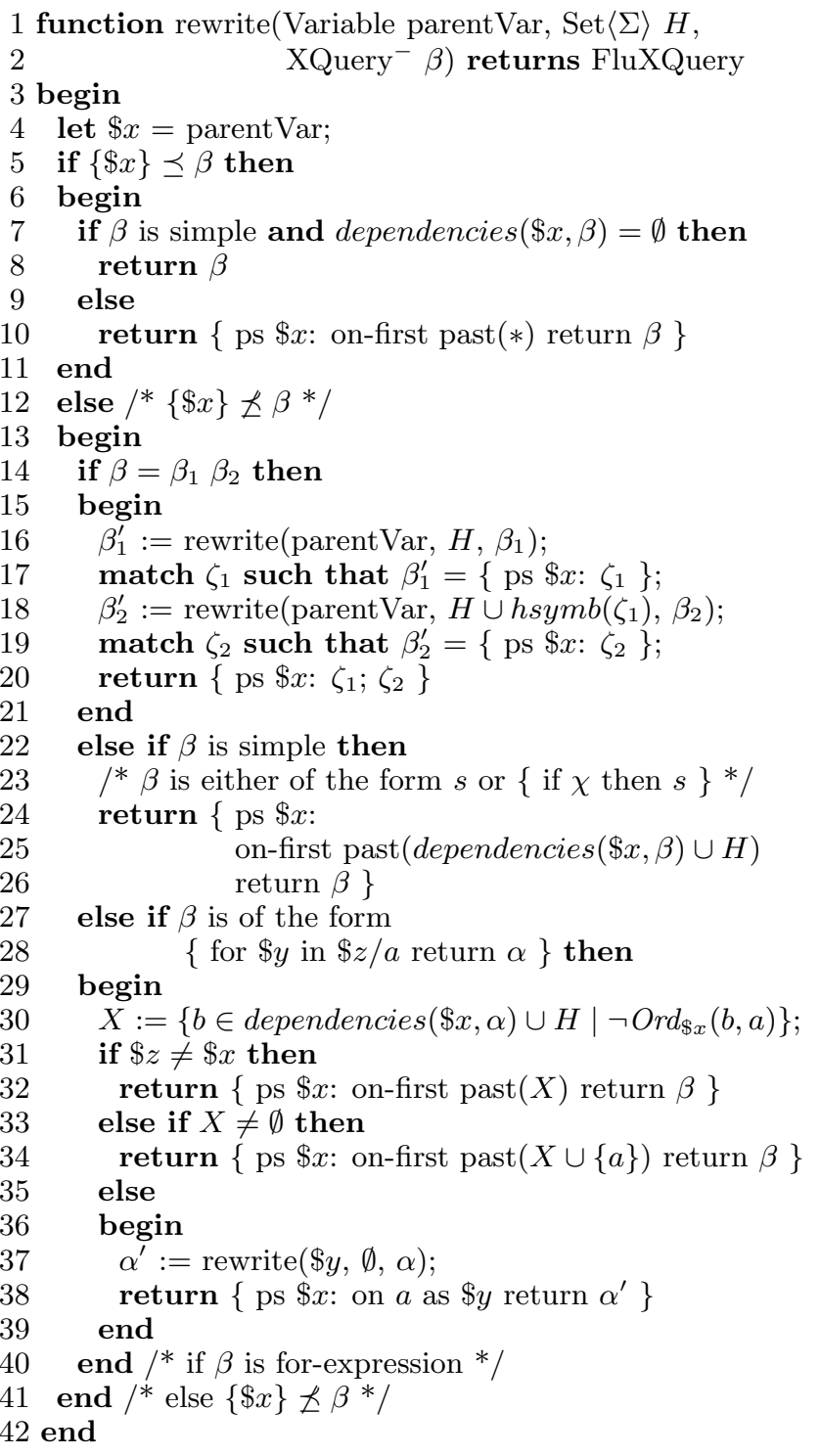

Figure 2: Algorithm for rewriting XQuery ${ }^{-}$into FluX.

query $Q$, we obtain the corresponding FluX query as "rewrite $(\$ R 00 \mathrm{~T}, \emptyset, Q)$ ". Some example runs of this algorithm are given in Section 4.3 below. The goals in the design of the algorithm were to produce a FluX query which (1) is safe w.r.t. the given DTD, (2) is equivalent to the input XQuery, and (3) minimizes the amount of buffering needed for evaluating the query in an XML document.

To meet goals (1) and (2), e.g. the particular order of the if-statements in the algorithm (lines 5, 14, $22,27)$ is crucial. Also, a set $H$ of handler symbols must be passed on in recursive calls of the algorithm, because otherwise the resulting FluX query would not be safe. One important construct for meeting goal (3) is the case distinction in lines 31-39, where an "on" handler is created provided that this is safe, and an "on-first" handler is created otherwise.

Theorem 4.3 Given a DTD $D$ and a normalized
$X$ uery $^{-}$query $Q$, "rewrite(\$ROOT, $\left.\emptyset, Q\right)$ " runs in time $O\left(|D|^{3}+|Q|^{2}\right)$ and produces a safe FluX query that is equivalent to $Q$ on all XML documents compliant with the given DTD.

Our algorithm performs only a single traversal of the query tree. Runtime $O\left(|Q|^{2}\right)$ is mainly caused by the need to compute dependencies. Note that the resulting FluX query is in normal form.

\subsection{Examples}

We now discuss the effect of our rewrite algorithm on sample queries from the XQuery Use Cases [18]. ${ }^{2}$

Example 4.4 ([18], XMP, Q2) Let us consider the XQuery $Q_{2}$ from the XQuery Use Cases [18], which creates a flat list of all the title-author pairs, with each pair enclosed in a result element. Due to space limitations we omit $Q_{2}$ here and only give its normalization $Q_{2}^{\prime}$ (which is very similar to the original XQuery $\left.Q_{2}\right)$ :

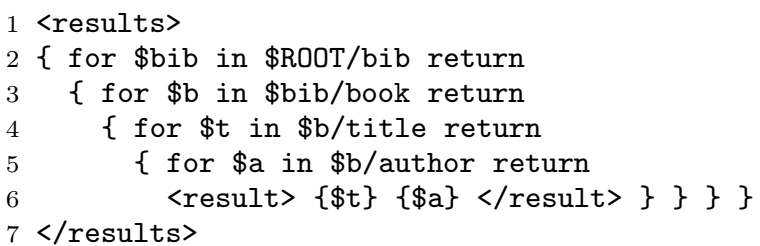

When given a DTD that does not impose any order constraints on title and author, e.g., the first DTD from Section 1, then "rewrite $\left(\$ R 00 \mathrm{~T}, \emptyset, Q_{2}^{\prime}\right)$ " proceeds as follows: First, $Q_{2}^{\prime}$ is decomposed into two subexpressions $\beta_{1}$, consisting of line 1 , and $\beta_{2}$, consisting of lines $2-7$. Then, the rewrite algorithm is recursively called for $\beta_{1}$ and for $\beta_{2}$. As $\beta_{1}$ is simple, the call for $\beta_{1}$ produces the result

\{ps \$ROOT: on-first past() return <results> \}

The call for $\beta_{2}$ decomposes $\beta_{2}$ into two subexpressions $\beta_{21}$, consisting of lines $2-6$, and $\beta_{22}$, consisting of line 7 of $Q_{2}^{\prime}$. The recursive call "rewrite(\$ROOT, $\left.\emptyset, \beta_{21}\right)$ " then executes lines $36-39$ of the algorithm in Figure 2, because $\beta_{21}$ is a for-loop with parent variable \$ROOT and associated set $X=X_{\beta_{21}}=\emptyset$. That is, the result

\{ps \$ROOT: on bib as \$bib return $\left.\alpha_{1}^{\prime}\right\}$

is produced, where $\alpha_{1}^{\prime}$ is the result produced by the recursive function call "rewrite $\left(\$ \mathrm{bib}, \emptyset, \alpha_{1}\right)$ ", for the subquery $\alpha_{1}$ of $Q_{2}^{\prime}$ in lines $3-6$. This recursive call for $\alpha_{1}$ again executes lines 36-39 of the algorithm, producing the expression $\alpha_{1}^{\prime}=$

\section{\{ps \$bib: on book as \$b return $\left.\alpha_{2}^{\prime}\right\}$}

where $\alpha_{2}^{\prime}$ is the result of "rewrite $\left(\$ \mathrm{~b}, \emptyset, \alpha_{2}\right)$ " for the subquery $\alpha_{2}$ of $Q_{2}^{\prime}$ in lines $4-6$. As $\alpha_{2}$ is a

\footnotetext{
${ }^{2}$ We rewrite the queries to work without attributes.
} 
for-loop with parent variable $\$ \mathrm{~b}$ and associated set $X=X_{\alpha_{2}}=\{$ author $\}$, in this call line 34 of the algorithm is executed, producing the expression $\alpha_{2}^{\prime}=$

\{ps \$b: on-first past(author,title) return $\alpha_{2}$ \}

All in all, "rewrite $\left(\$ \mathrm{ROOT}, \emptyset, Q_{2}^{\prime}\right)$ " returns the following FluX query $F_{2}$ :

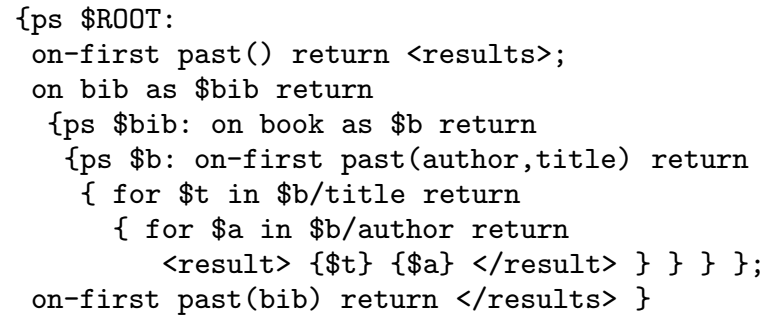

We will refer to the " $\{\mathrm{ps} \$ \mathrm{~b} \cdots\}$ "-expression in lines 5-8 of $F_{2}$ as $\alpha_{2}^{\prime}$. When evaluating the query $F_{2}$ on an XML document, the XQuery inside $\alpha_{2}^{\prime}$ will be evaluated once all author and all title nodes have been encountered and buffered.

Let us now consider the case where we are given a DTD with the production

\section{$<$ !ELEMENT book (author*,title*)>}

where the order constraint $\operatorname{Ord}_{\mathrm{book}}$ (author, title) is met. While running "rewrite $\left(\$ \mathrm{ROOT}, \emptyset, Q_{2}^{\prime}\right)$ " we now encounter the situation where $X=X_{\alpha_{2}}=\emptyset$ (rather than $\{$ author $\}$, as with the previous DTD). Therefore, when processing the recursive call "rewrite $\left(\$ \mathrm{~b}, \emptyset, \alpha_{2}\right)$ ", now lines 36-39 of the algorithm are executed, eventually producing the following result $\alpha_{2}^{\prime \prime}=$

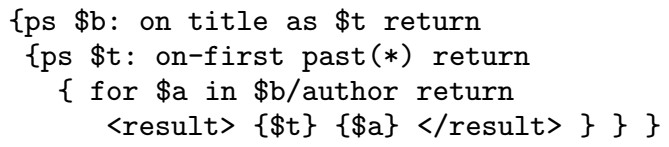

Now, "rewrite $\left(\$ R 00 \mathrm{~T}, \emptyset, Q_{2}^{\prime}\right)$ " yields query $F_{2}^{\prime}$ differing from $F_{2}$ in the lines $5-8$, which must be replaced by the above expression $\alpha_{2}^{\prime \prime}$.

When evaluating $F_{2}^{\prime}$ on an XML document compliant with the second DTD, all author nodes arrive before title nodes and are buffered. Encountering a title node in the input stream invokes the following actions: The value of that particular node is buffered, i.e., "on-first past(*)" delays the execution until the complete title node has been seen. Then, we iterate over the buffer containing all collected author nodes, each time writing the buffered title and the current author to the output. In contrast to the worstcase scenario above, we only buffer one title at a time in addition to the list of all authors. If there is more than one title, this strategy is clearly preferable.

We next demonstrate that conditional for-loops are optimized correspondingly.

Example 4.5 ([18], XMP, Q1) Let us consider the query $Q_{1}$ and its normalization $Q_{1}^{\prime}$ from Example 4.2.
Given a DTD that does not impose any order constraints, e.g., the DTD

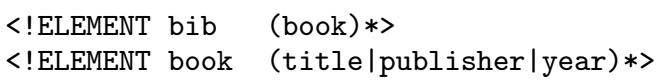

the function call "rewrite $\left(\$ \mathrm{R} 00 \mathrm{~T}, \emptyset, Q_{1}^{\prime}\right)$ " rewrites $Q_{1}^{\prime}$ into the following FluX query $F_{1}$ :

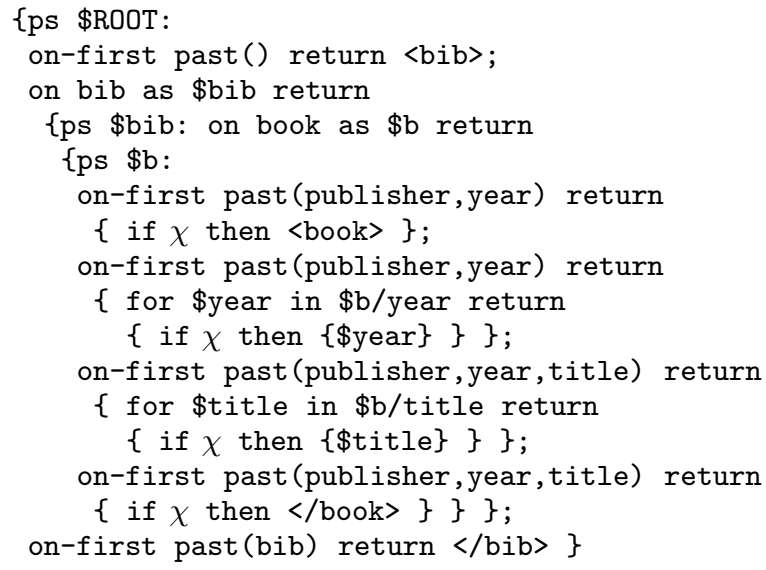

The "on-first" handler in lines 11-13 delays query execution until all title nodes have been buffered and all publisher and year nodes have been seen.

When given a different DTD, ensuring that both $\operatorname{Ord}_{\text {book }}\left(\right.$ year,title) and $\operatorname{Ord}_{\text {book }}$ (publisher,title) hold, the title nodes can be processed in the streaming fashion. The query $F_{1}^{\prime}$ produced by "rewrite $\left(\$ \mathrm{ROOT}, \emptyset, Q_{1}^{\prime}\right)$ " with this new DTD differs from the above query $F_{1}$ in the subexpression in lines 11-13 which must be replaced by

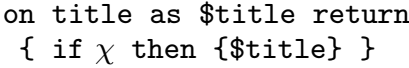

Consequently, titles will not be buffered at all during evaluation of this query.

Our rewrite algorithm is well capable of optimizing joins over two or more join predicates, as is demonstrated in the following example which is not part of the XQuery Use Cases.

Example 4.6 We remain in the bibliography domain and consider documents compliant with the DTD

$<$ !ELEMENT bib (book|article)*>

$<$ !ELEMENT book (title, (author+|editor+),publisher)> $<$ !ELEMENT article (title, author+,journal)>

The following XQuery $Q_{3}$ retrieves those authors of articles which are coauthored by people who have also edited books:

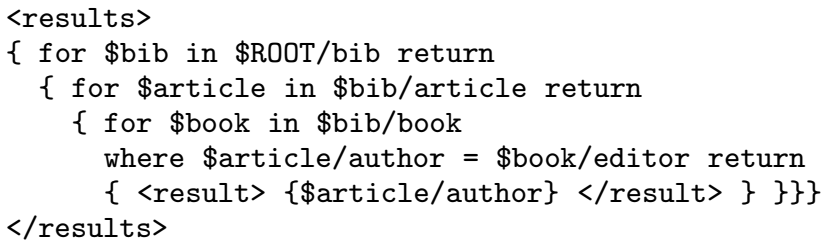


For the remainder of this example, we abbreviate the join-condition comparing the authors of articles with the editors of books by $\chi$. Normalization yields the following query $Q_{3}^{\prime}$ :

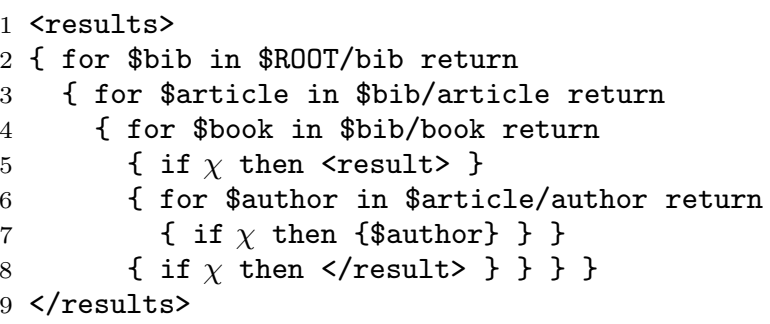

When executing "rewrite $\left(\$ \mathrm{ROOT}, \emptyset, Q_{3}^{\prime}\right)$ " with the DTD above, a recursive call "rewrite $(\$ \mathrm{bib}, \emptyset, \beta)$ " is eventually invoked for the subexpression $\beta$ of $Q_{3}^{\prime}$ in lines 3-8. As $\beta$ is a for-loop with parent variable $\$ \mathrm{bib}$ and associated set $X=X_{\beta}=\{$ book $\} \neq \emptyset$, line 34 of the algorithm is executed, returning an expression of the form \{ps \$bib: on-first past (book, article) ... \}. That is, as no order constraint between article and book holds, an on-first handler ensures that all articles and books will be buffered.

Altogether, "rewrite $\left(\$ \mathrm{R} 0 \mathrm{OT}, \emptyset, Q_{3}^{\prime}\right)$ " produces the following FluX query $F_{3}$, where $\alpha$ is used as abbreviation for the for-loop over books in lines 4-8 of $Q_{3}^{\prime}$ :

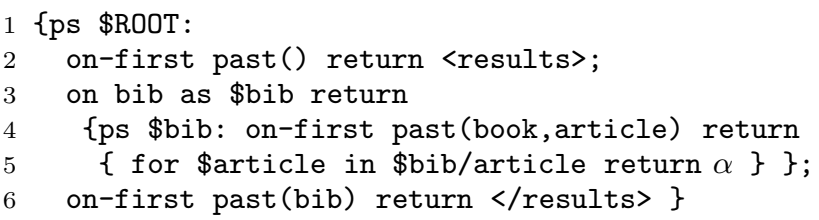

When given a different DTD which imposes an order on books and articles, e.g. by the following production

\section{$<$ !ELEMENT bib (book*,article*)>}

we can evaluate $Q_{3}^{\prime}$ by buffering only book nodes but processing article nodes in a streaming fashion.

Indeed, when executing "rewrite(\$ROOT, $\left.\emptyset, Q_{3}^{\prime}\right)$ " with this new DTD, we eventually encounter the situation where set $X=X_{\beta}=\emptyset$, and therefore, lines 36-39 (rather than line 34 , as with the previous DTD) are executed. Altogether, the FluX query $F_{3}^{\prime}$ produced now, differs from the above query $F_{3}$ in the subexpression in lines $4-5$, which must be replaced by

4 \{ps \$bib: on article as \$article return

5 \{ps \$article: on-first past(author) return $\alpha$ \}\};

As all book nodes will have arrived before an article node can be encountered, data from books is available in buffers once the first article node is being read. When processing the children of an article node, we first buffer all author nodes before the query can be evaluated for the current article.

During the evaluation of $F_{3}^{\prime}$, we therefore only buffer the authors of a single article in addition to the data already stored on books, whereas the evaluation of $F_{3}$ requires the authors of all articles to be buffered.

\section{Implementation}

In this section, we discuss our implementation of a query engine for evaluating FluX queries obtained from $\mathrm{XQuery}^{-}$by the rewriting algorithm of the previous section.

We focus on the allocation of buffers and their use during query evaluation. Given a FluX query, we statically infer the buffers which are actually necessary in order to avoid superfluous buffering. Our prefiltering techniques generalize those of [14] to the scenario where certain parts of the input do not need to be buffered - even though they are used by the query because they can be processed on-the-fly.

Buffers are implemented as lists of SAX events. The events stored in a buffer represent well-formed XML in the sense that start-element events and end-element events are properly nested within each other. This renders data read from (a stream replayed from) a buffer indistinguishable from data read from the input stream. In our implementation, we employ the same set of operators for handling both events originating from streams and from buffers. ${ }^{3}$

In the following, we say that a FluX query is in normal form if all of its (maximal) $\mathrm{XQuery}^{-}$subexpressions are in normal form.

Let $Q$ be a safe FluX query in normal form. The FluX query engine identifies all nodes that must be stored in buffers, i.e. all nodes compared in join conditions, the roots of buffered subtrees that are output, and buffered nodes over which for-loops iterate.

More formally, let $\alpha$ be an $\mathrm{XQuery}^{-}$subexpression of $Q$. We define $\Pi(\$ r, \alpha)$, the set of all buffered paths in $\alpha$ starting with variable $\$ r$, as $\Pi(\$ r, \epsilon)=\Pi(\$ r, s)=$ $\emptyset, \Pi(\$ r,\{\$ r\})=\{\$ r\}, \Pi(\$ r, \alpha \beta)=\Pi(\$ r, \alpha) \cup \Pi(\$ r, \beta)$,

$$
\begin{aligned}
& \Pi(\$ r, \text { for } \$ x \text { in } \$ y / a \text { return } \alpha\})= \\
& \Pi(\$ r, \alpha) \cup\{\$ r / a \mid \$ y=\$ r \text { and } \Pi(\$ x, \alpha)=\emptyset\} \\
& \cup\{\$ r / a / w \mid \$ y=\$ r \text { and } \$ x / w \in \Pi(\$ x, \alpha)\},
\end{aligned}
$$

and $\Pi(\$ r,\{$ if $\chi$ then $\alpha\})=\Pi(\$ r, \alpha) \cup\{\$ r / \pi$ $\chi$ contains an atomic condition $\$ r / \pi \operatorname{RelOp} \$ y / \pi^{\prime}$ or $\left.\$ y / \pi^{\prime} \operatorname{RelOp} \$ r / \pi\right\}$.

For a variable $\$ r$ and a safe FluX query $Q$ in normal form, we now define $\Pi(\$ r)$ as the union of all $\Pi(\$ r, \alpha)$ s.t. $\alpha$ is a maximal XQuery ${ }^{-}$subexpression of $Q$.

Let $\mathcal{T}(\$ r)$ be the prefix tree constructed by merging all paths from $\Pi(\$ r)$. Intuitively, the prefix tree defines a projection of the input document, as it describes which parts of the input tree will be buffered.

We optimize the prefix tree in order to restrict the amount of data being buffered. Let $\mathcal{T}^{m}(\$ r)$ be the tree obtained from $\mathcal{T}(\$ r)$ by marking each node $v$ if $v$ either occurs in a join condition or the entire subtree rooted at node $v$ is output and must therefore be buffered. For unmarked nodes in $\mathcal{T}^{m}(\$ r)$, we merely store the SAX events for the opening and the closing tag.

\footnotetext{
${ }^{3}$ Thus, physical query evaluation proceeds in a way similar to that followed in XQRL [9].
} 


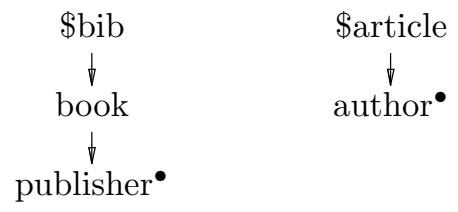

Figure 3: Buffer trees of variables $\$$ bib and $\$$ article.

Clearly, if a node is marked and we buffer it together with its subtree, we also buffer the subtrees of any descendant nodes at the same time. Thus we only buffer the data of the topmost marked nodes in $\mathcal{T}^{m}(\$ r)$. For example, if we need to buffer two subtrees reachable by paths $\pi$ and $\pi^{\prime}$ respectively, where $\pi$ is a prefix of $\pi^{\prime}$, we restrict ourselves to buffering the subtree identified by $\pi$. Let $\mathcal{T}^{p}(\$ r)$ be the pruned prefix tree obtained from $\mathcal{T}^{m}(\$ r)$ by successively removing a subtree rooted at node $v^{\prime}$ if an ancestor node $v$ is marked. We refer to $\mathcal{T}^{p}(\$ r)$ as a buffer tree.

W.l.o.g., below we will assume that variables in queries are used uniquely, i.e., each variable name is bound at most at one place in the query. For a safe FluX query $Q$ in normal form, let $X$ be the set of variables that are free in maximal XQuery ${ }^{-}$subexpressions of $Q$. The variables in $X$ are precisely those for which we will later define buffers.

Example 5.1 The following FluX query selects all book publishers whose $\mathrm{CEO}$ has published articles.

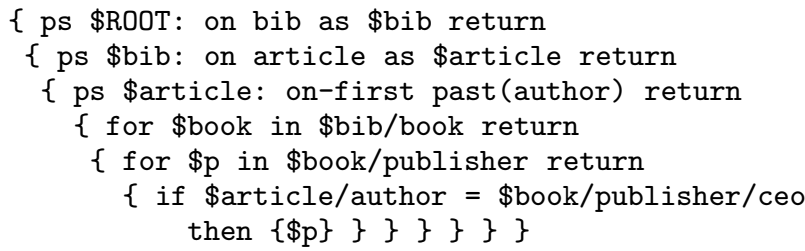

Here, $X=\{\$$ bib, $\$$ article $\}$ and we compute the sets of buffer paths for each variable in $X$ :

$$
\begin{aligned}
\Pi(\text { \$bib })= & \{\text { \$bib } / \text { book } / \text { publisher } / \text { ceo }, \\
& \text { \$bib } / \text { book } / \text { publisher }\} \\
\Pi(\text { \$article })= & \{\text { \$article/author }\}
\end{aligned}
$$

We construct a buffer tree for each variable in $X$ with a nonempty set of buffer paths. Here, we obtain the trees shown in Figure 3 (the bullet denotes a marked node). Note that the leaf node ceo has been pruned off the buffer tree of variable $\$$ bib.

We evaluate a safe FluX query $Q$ in normal form as follows. We compute $X$ and construct the buffer tree $\mathcal{T}^{p}(\$ r, Q)$ for each variable $\$ r \in X$.

We further associate an evaluator function with each variable $\$ r$ in $X$. When variable $\$ r$ is bound to a node $v$ of the incoming XML tree, then the evaluator Eval_\$r is responsible for handling all events generated while processing the children of $v$.

We define a buffer buffer_\$r for variable $\$ r$ in the evaluator which calls Eval_\$r. This buffer is initialized on entering the scope of $\$ r$ and freed on re-entering it.
The buffer tree of $\$ r$ can be considered a schema for all events stored in buffer buffer_\$r. At the same time, the buffer tree determines how the set of evaluators is to be extended such that all buffers are correctly filled.

For a node $v$ in buffer tree $\mathcal{T}^{p}(\$ r)$, reachable by path $\$ r / a_{1} / \ldots / a_{n}$, we implement the corresponding buffering strategy in a set of evaluators. Starting with Eval_\$r and $a_{1}$, we successively extend the evaluators responsible for handling the children of a node labeled $a_{i}$ such that the events for the opening and closing tag of the respective node will be added to buffer_\$r. In cases where no such evaluator exists, we introduce new variables and evaluators accordingly. As there is at most one case statement in an evaluator for an "on a" event under a given node label "a", it is clear where corresponding commands are to be introduced. In case $a_{n}$ is marked, we insert the respective code for adding all events corresponding to node $a_{n}$ and its subtree to buffer_\$r.

Example 5.2 Consider query $F_{3}^{\prime}$ of Example 4.6. The set of buffer trees for this query is as in Figure 3 with the publisher node replaced by an editor node. Its FluX evaluation strategy is as follows.

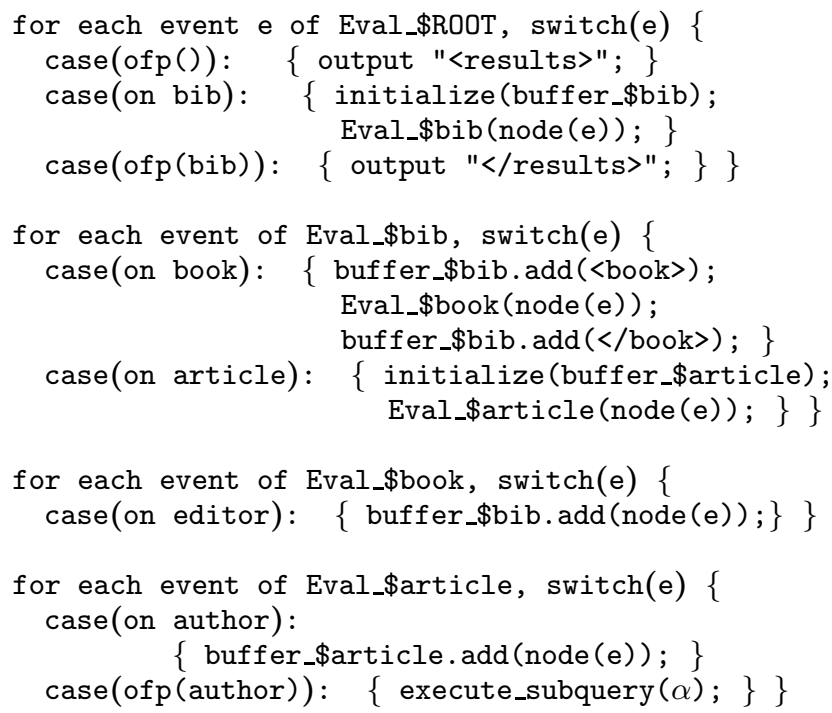

At the beginning of the stream, the evaluator of variable \$ROOT handles the event on-first past(), denoted ofp() above, by writing the opening tag $\langle$ results $>$ to the output. Correspondingly, the event of $(\mathrm{bib}$ ) signals the end of the stream and the closing tag $</$ results $>$ is output.

Yet when processing the SAX event for the opening tag of root node bib, the buffer associated with variable $\$ b i b$ is initialized and the evaluator for $\$ b i b$ takes over to handle all events generated while parsing the children of bib.

Book nodes arriving on the stream are stored in the buffer of variable $\$ b i b$, while editor nodes are buffered by the evaluator for variable \$book together with their complete subtrees. As with all safe FluX queries, we may rely on the fact that buffer 


\begin{tabular}{|cr||c|c|c|}
\hline & & FluX & Galax & AnonX \\
\hline \hline \multirow{2}{*}{$Q_{1}$} & $5 \mathrm{M}$ & $2.1 \mathrm{~s} / 0$ & $13.4 \mathrm{~s} / 37 \mathrm{M}$ & $3.4 \mathrm{~s}$ \\
& $10 \mathrm{M}$ & $2.8 \mathrm{~s} / 0$ & $29.8 \mathrm{~s} / 83 \mathrm{M}$ & $6.7 \mathrm{~s}$ \\
& $50 \mathrm{M}$ & $7.8 \mathrm{~s} / 0$ & $-/>500 \mathrm{M}$ & $38.3 \mathrm{~s}$ \\
& $100 \mathrm{M}$ & $14.0 \mathrm{~s} / 0$ & $-/>500 \mathrm{M}$ & - \\
\hline \multirow{2}{*}{$Q_{8}$} & $5 \mathrm{M}$ & $6.8 \mathrm{~s} / 1.54 \mathrm{M}$ & $296.9 \mathrm{~s} / 50 \mathrm{M}$ & $143.8 \mathrm{~s}$ \\
& $10 \mathrm{M}$ & $17.2 \mathrm{~s} / 3.16 \mathrm{M}$ & $1498.3 \mathrm{~s} / 100 \mathrm{M}$ & $534.8 \mathrm{~s}$ \\
& $50 \mathrm{M}$ & $357.8 \mathrm{~s} / 16.00 \mathrm{M}$ & $-/>500 \mathrm{M}$ & - \\
& $100 \mathrm{M}$ & $11566.9 \mathrm{~s} / 32.25 \mathrm{M}$ & $-/>500 \mathrm{M}$ & - \\
\hline \multirow{2}{*}{$Q_{11}$} & $5 \mathrm{M}$ & $5.6 \mathrm{~s} / 374 \mathrm{k}$ & $277.0 \mathrm{~s} / 50 \mathrm{M}$ & $\mathrm{n} / \mathrm{a}$ \\
& $10 \mathrm{M}$ & $11.4 \mathrm{~s} / 741 \mathrm{k}$ & $1663.7 \mathrm{~s} / 100 \mathrm{M}$ & $\mathrm{n} / \mathrm{a}$ \\
& $50 \mathrm{M}$ & $170.8 \mathrm{~s} / 3.64 \mathrm{M}$ & $-/>500 \mathrm{M}$ & $\mathrm{n} / \mathrm{a}$ \\
& $100 \mathrm{M}$ & $626.8 \mathrm{~s} / 7.27 \mathrm{M}$ & $-/>500 \mathrm{M}$ & $\mathrm{n} / \mathrm{a}$ \\
\hline \multirow{2}{*}{$Q_{13}$} & $5 \mathrm{M}$ & $2.2 \mathrm{~s} / 0$ & $12.8 \mathrm{~s} / 38 \mathrm{M}$ & $3.0 \mathrm{~s}$ \\
& $10 \mathrm{M}$ & $3.1 \mathrm{~s} / 0$ & $27.2 \mathrm{~s} / 73 \mathrm{M}$ & $5.2 \mathrm{~s}$ \\
& $50 \mathrm{M}$ & $7.9 \mathrm{~s} / 0$ & $230.1 \mathrm{~s} / 344 \mathrm{M}$ & $88.0 \mathrm{~s}$ \\
& $100 \mathrm{M}$ & $13.9 \mathrm{~s} / 0$ & $-/>500 \mathrm{M}$ & - \\
\hline \multirow{2}{*}{$Q_{20}$} & $5 \mathrm{M}$ & $2.8 \mathrm{~s} / 4.66 \mathrm{k}$ & $13.2 \mathrm{~s} / 36 \mathrm{M}$ & $2.5 \mathrm{~s}$ \\
& $10 \mathrm{M}$ & $3.4 \mathrm{~s} / 5.18 \mathrm{k}$ & $29.7 \mathrm{~s} / 80 \mathrm{M}$ & $6.2 \mathrm{~s}$ \\
& $50 \mathrm{M}$ & $8.7 \mathrm{~s} / 7.01 \mathrm{k}$ & $-/>500 \mathrm{M}$ & $151.9 \mathrm{~s}$ \\
& $100 \mathrm{M}$ & $15.4 \mathrm{~s} / 7.02 \mathrm{k}$ & $-/>500 \mathrm{M}$ & - \\
\hline
\end{tabular}

Figure 4: Benchmark results.

buffer_\$bib is filled by the time we encounter the first article node. The buffer is not freed until the complete subtree under the node bound to variable $\$$ bib has been parsed.

Processing the children of an article node, any authors are first buffered in buffer_\$article until the event ofp (author) guarantees that the subquery $\alpha$ of $F_{3}^{\prime}$ can be executed correctly.

Join conditions are handled similarly, by buffering both constituent paths of the condition. Simple conditions comparing a path with a constant can be evaluated on the fly while reading the paths, so only a Boolean flag is required, which has to be appropriately initialized upon entering the relevant variable scope.

\section{Experiments}

In order to assess the merits of the approach presented in this paper, we have experimentally evaluated our prototype query engine implemented in JAVA using a number of queries on data obtained using the XMark benchmark generator.

Our implementation supports the XQuery ${ }^{-}$fragment as defined in Section 3. We took selected queries of the XMark benchmark and, as XQuery ${ }^{-}$does not include certain features that are used in these queries, adapted them correspondingly. In detail, attributes were converted into subelements of their parent element in our tests (the XMark DTD was adjusted accordingly). Occurrences of the XPath step text() were replaced by $\{\$ \mathrm{x}\}$ expressions that print the whole element instead. We eliminated the count $(\$ x)$ aggregations by again outputting $\$ x$ instead. XMark queries $1,8,11$, and 13 were adjusted as sketched above. We extracted the last FLWR subexpression of original query 20 (which computes persons whose income is not available) for our novel query 20. The queries thus obtained can be found in full in [12].

We used data generated by the XMark xmlgen data generation tool (V. 0.96) of the sizes $5 \mathrm{MB}, 10 \mathrm{MB}$, $50 \mathrm{MB}$, and $100 \mathrm{MB}$ as input data. All tests were performed with the SUN JDK 1.4.2_03 and the built-in SAX parser on an AMD Athlon XP $2000+(1.67 \mathrm{GHz})$ with 512MB RAM running Linux (gentoo linux using kernel 2.6). Our query engine was implemented precisely as described in this paper. As a reference implementation the Galax query engine (V. 0.3.1) was employed with projection turned on [14]. The performance of query evaluation was studied by measuring the execution time ${ }^{4}$ (in seconds) and maximum memory consumption (in bytes) of each engine. The memory and CPU usage of both query engines were measured by internal monitoring functions (excluding the fixed memory consumption of the Java Virtual Machine).

To give a broader overview over the performance of our approach we evaluated our queries additionally with a commercial XQuery system of a major company that has to remain anonymous and will be called AnonX below. Unfortunately, we could not determine the exact memory consumption for this system. Hence, we only state its execution time. As AnonX was not able to parse Query 11, we are not able to list the execution time.

Figure 4 shows the results of our experiments. To evaluate most queries with input greater than $10 \mathrm{MB}$, Galax needed more than 500MB of main memory after running for a few minutes (which caused the system to start swapping). These runs were aborted. Obviously, our prototype engine clearly outperforms Galax with respect to both execution time and memory consumption. Queries 1 and 13 are evaluated on-the-fly without any buffering because of the order constraints imposed by the DTD. Query 20 has to buffer only a single element at a time, which leads to very low memory consumption in comparison to the traditional approach. Queries 8 and 11 perform a join on two subtrees (i.e. of people and closed_auction resp. open_auction) and therefore inevitably have to buffer elements. Nevertheless, due to our effective projection scheme only a small fraction of the original data is buffered. The rapid increase in execution time is due to the fact that we compute joins by naive nested loops at the moment. (We will work on this orthogonal but vital issue in the future.)

The comparison of the execution times to AnonX again shows the competitiveness of our query engine. AnonX ran out of memory processing queries marked by "-" (the maximum heap size of the Java VM was set to $512 \mathrm{MB}$ in both cases) and hence did not give any results in this case.

Altogether, our optimization approach seems to perform very well with respect to execution time, max-

\footnotetext{
${ }^{4}$ The times taken for query rewriting were negligible and are not reported separately in our experiments.
} 
imum memory consumption, and the maximum size of XML documents that can be processed.

\section{Discussion and Conclusions}

Main memory is probably the most critical resource in (streamed) query processing. Keeping main memory consumption low is vital to scalability and has - indirectly - a great impact on query engine performance in terms of running time.

The main contribution of this paper is the FluX language together with an algorithm for automatically translating a significant fragment of XQuery into equivalent FluX queries. FluX - while intended as an internal representation format for queries rather than a language for end-users - provides a strong intuition for buffer-conscious query processing on structured data streams. The algorithm uses schema information to schedule FluX queries so as to reduce the use of buffers.

As evidenced by our experiments, our approach indeed dramatically increases the scalability of main memory XQuery engines, even though we think we are not yet close to exhausting this approach, neither with respect to run-time buffer management and query processing nor query optimization.

In particular, further constraints such as cardinality constraints derived from the DTD, telling, e.g., that a book node has at most one publisher child (let this be denoted by publisher $\in \|_{\text {book }}^{\leq 1}$ ), could be used to simplify XQueries before they are rewritten into FluX using rewrite rules such as

$$
\frac{\left\{\begin{array}{l}
\text { for } \$ x \text { in } \$ r / a \text { return } \alpha \\
\text { for } \$ x \text { in } \$ r / a \text { return } \beta\}
\end{array}\right.}{\{\text { for } \$ x \text { in } \$ r / a \text { return } \alpha \beta\}} \quad\left(a \in \|_{\$ r}^{\leq 1}\right)
$$

which can form the basis of algebraic query optimization for buffer minimization.

Sequences of for-loops iterating over singletons are a natural product of the normalization process that we have described. For example, the query

\section{$\{$ for $\$ \mathrm{~b}$ in $\$ \mathrm{ROOT} /$ book return \\ $\{\$ \mathrm{~b} /$ publisher/name $\}$ \{b/publisher/address $\}$ \}}

uses a sequence of two loops over publisher in its normal form, which can be rewritten into one using the rule above. By first merging for-loops, it is often possible to obtain FluX queries that require no buffering at all, while two subsequent loops over the same path generally cause that path to be buffered.

Another important optimization is to push ifexpressions - which we have moved down the query tree to obtain our normal form - back "up" the expression tree as soon as the other simplifications have been realized.

\section{References}

[1] S. Abiteboul, P. Buneman, and D. Suciu. Data on the Web. Morgan Kaufmann Publishers, 2000.
[2] M. Altinel and M. Franklin. "Efficient Filtering of XML Documents for Selective Dissemination of Information". In Proc. VLDB 2000, pages 53-64, Cairo, Egypt, 2000.

[3] A. Brüggemann-Klein and D. Wood. "OneUnambiguous Regular Languages". Information and Computation, 142(2):182-206, 1998.

[4] P. Buneman, M. Grohe, and C. Koch. "Path Queries on Compressed XML". In Proc. VLDB 2003, pages 141-152, 2003.

[5] C. Y. Chan, P. Felber, M. N. Garofalakis, and R. Rastogi. "Efficient Filtering of XML Documents with XPath Expressions". In Proc. ICDE 2002, San Jose, California, USA, February 26-March 12002.

[6] A. Deutsch and V. Tannen. "Reformulation of XML Queries and Constraints". In Proc. ICDT'03, 2003.

[7] L. Fegaras, D. Levine, S. Bose, and V. Chaluvadi. "Query Processing of Streamed XML Data". In Proc. CIKM 2002, pages 126-133, 2002.

[8] M. Fernandez, J. Siméon, and P. Wadler. "A Semimonad for Semi-structured Data". In Proc. ICDT'01, pages 263-300, 2001.

[9] D. Florescu, C. Hillery, D. Kossmann, P. Lucas, F. Riccardi, T. Westmann, M. J. Carey, A. Sundararajan, and G. Agrawal. "The BEA/XQRL Streaming XQuery Processor". In Proc. VLDB 2003, pages 9971008, 2003.

[10] T. J. Green, G. Miklau, M. Onizuka, and D. Suciu. "Processing XML Streams with Deterministic Automata". In Proc. ICDT'03, 2003.

[11] A. K. Gupta and D. Suciu. "Stream Processing of XPath Queries with Predicates". In SIGMOD Conference, pages 419-430, 2003.

[12] C. Koch, S. Scherzinger, N. Schweikardt, and B. Stegmaier. "Schema-based Scheduling of Event Processors and Buffer Minimization for Queries on Structured Data Streams". Technical Report cs.DB/0406016, CoRR, 2004. http://arxiv.org/abs/cs.DB/0406016.

[13] B. Ludäscher, P. Mukhopadhyay, and Y. Papakonstantinou. "A Transducer-Based XML Query Processor". In Proc. VLDB 2002, pages 227-238, 2002.

[14] A. Marian and J. Siméon. "Projecting XML Documents". In Proc. VLDB 2003, pages 213-224, 2003.

[15] M. Murata, D. Lee, and M. Mani. "Taxonomy of XML Schema Languages using Formal Language Theory". In Extreme Markup Languages, Montreal, Canada, Aug. 2001.

[16] D. Olteanu, T. Kiesling, and F. Bry. "An Evaluation of Regular Path Expressions with Qualifiers against XML Streams". In Proc. ICDE 2003, Bangalore, Mar. 2003. Poster Session.

[17] World Wide Web Consortium. "XQuery 1.0 and XPath 2.0 Formal Semantics. W3C Working Draft (Aug. 16th 2002), 2002. http://www.w3.org/TR/query-algebra/.

[18] "XML Query Use Cases. W3C Working Draft 02 May 2003", 2003. http://www.w3.org/TR/xmlquery-usecases/. 